\title{
Data Analysis with Pandas
}

\section{Maria Cristina ENACHE ${ }^{\star}$}

\begin{tabular}{l}
\hline \multicolumn{1}{c}{ A R T I C L E I N F O } \\
\hline Article history: \\
Accepted May 2019 \\
Available online August 2019 \\
\hline JEL Classification \\
K22, M21 \\
Keywords: \\
Data analysis, \\
Python \\
\end{tabular}

\begin{abstract}
A B S T R A C T
Data analysis is the process by which data is cleaned, analyzed and modeled using tools. This is used for marketing strategies to get the desired business result. Not many companies have realized the importance of using large data analytics to maximize their profit. Data analysis provides both the speed and accuracy of a business decision. It provides precision as well as a good statistical module and hi-tech tools to help and analyze data. Data analysis is the differentiator that gives companies a competitive edge over others. All data should make sense now. Data analysis makes numbers become real factors that, predictably, can make or destroy a company.
\end{abstract}

\section{Introduction}

Data analysis is the process of evaluating data using analytical and logical reasoning to examine each component of the data provided. This form of analysis is just one of the many stages that need to be completed when conducting a research experiment. Data from various sources is gathered, reviewed and then analyzed to form a sort of finding or conclusion. There are a variety of specific data analysis methods, some of which include data mining, text analytics, business information, and data views.

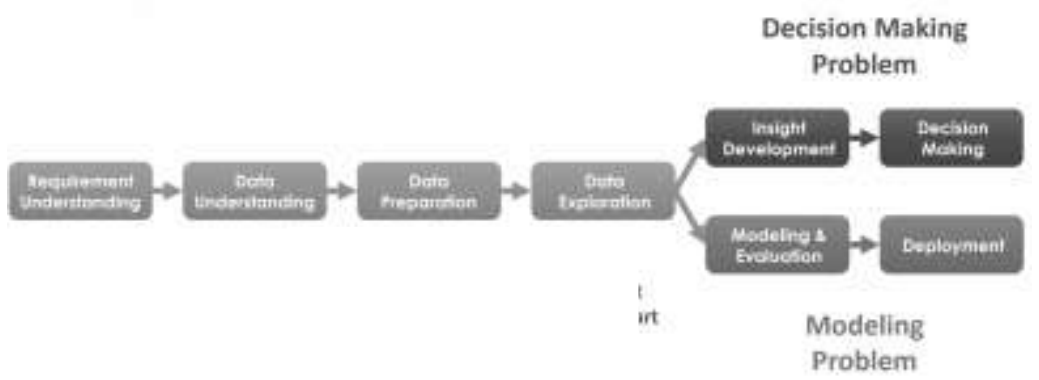

Figure 1 -Typical Process of Data Analytics

The data analysis steps are:

Descriptive Analysis: What Happens?

Predictive analysis: What could happen in the future?

Prescriptive Analytic: How should we respond to these potential future events?

- Descriptive Analysis are a preliminary data processing step that creates a summary of historical data to provide useful information and possibly to prepare the data for further analysis. Data extraction methods make it possible to identify patterns and relationships that would otherwise not be visible. Data query, reporting, and viewing can be applied to gain a better understanding.

- Diagnostic analysis is a deeper analysis of data to try to understand the causes of events and behaviors.

- The predictive analysis, which is used to identify future probabilities and trends, is said to provide information on what might happen in the future.

- $\quad$ Prescriptive Analytic are applied to try to identify the best results of the events, taking into account the parameters and to suggest decisional options to make the best use of a future opportunity or to mitigate future risks. 


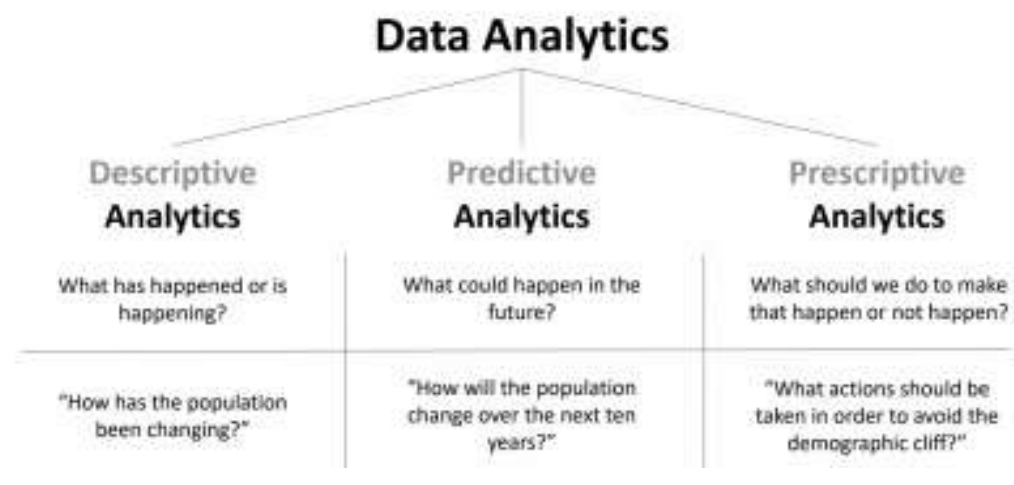

Figure 2 - Data analytics types

\section{Data Analysis vs. Data Analytics} data.

Data analytics is the process and methodology of analyzing data to draw meaningful insight from the

Data Analysis (DA) is the process of examining data sets to draw conclusions about the information they contain, increasingly with specialized systems and software. Data analysis technologies and techniques are widely used in the economy to enable organizations to make more informed decisions by scientists and researchers to check or review models, theories and scientific hypotheses. As a term, data analysis mainly refers to a wide range of applications, from basic business information (BI), online analytics reporting (OLAP) to various forms of advanced analysis. In this sense, it is similar in nature to business analysis, another umbrella term for data analysis approaches - with the difference that the latter is business-oriented, while data analyzes are more focused. In some cases, users use data analytics to specifically designate advanced analytics. Data analysis can help companies increase revenue, improve operational efficiency, optimize marketing campaigns, and strive for customer service, respond more quickly to emerging market trends, and gain competitive edge over rivals, all with the ultimate goal to increase business performance. Depending on the particular application, the data analyzed may consist either of historical records or of new information that has been processed for real-time analytical use. In addition, it may come from a mixture of internal systems and external data sources.

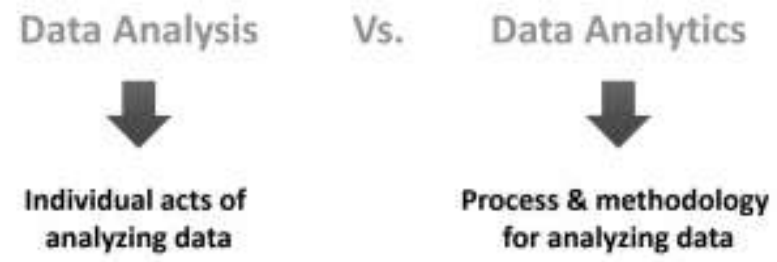

Figure 3 - Data analysis vs Data Analytics

\section{Big Data Vs. Data Analytics}

Big Data are data stored and processed in huge amounts due to cheaper storage media, faster processing methods, and better algorithms. Big Data has 4 main features:

- The first feature is volume - the data volume is growing, but it is not just the volume of data that is growing, the number of data sources is also growing.

- The second feature is spirit - data is created at increasing speeds and business requirements have grown and the world is also increasingly instrumental and interconnected.

- The third characteristic is the data variety - The variety of data presents an equally difficult challenge and traditional analysis methods only apply to structured information.

- The fourth characteristic is the trusted data - How can I act on this information if I'm not trusted. Establishing trust in the data that any company uses is a huge challenge with increasing data sources and types. 


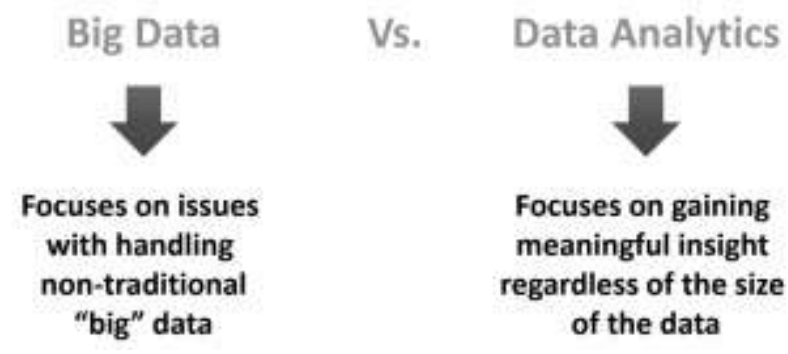

Figure 4 -Big Data vs Data Analytics

\section{Python and data analysis}

Python's popularity lies on two main pillars. One is that it is an easy-to-learn programming language designed to be highly readable, with a syntax quite clear and intuitive. And the second reason is its userfriendliness does not take away from its strength. Python can execute a variety of complex computations and is one of the most powerful programming languages preferred by specialists.

The Jupyter Notebook App is a server-client application that allows you to edit your code through a web browser. Language kernels are programs designed to read and execute code in a specific programming language, like Python, R, or Julia. The Jupyter installation always comes with an installed Python kernel, and the other kernels can be installed additionally. The Interfaces, where you can write code, represent the clients. An example of such a client is the web browser. The Jupyter server provides the environment where a client is matched with a corresponding languages kernel. In our case, we will focus on Python, and a web browser as a client. because is

Python as a Data Analytics Tool - The nature of Python makes it a perfect-fit for data analytics

- easy to learn

- readable s

- scalable

- extensive set of libraries

- easy integration with other apps

- active community \& ecosystem

Popular Python Data Analytics Libraries are

\begin{tabular}{|c|c|}
\hline Library & Usage \\
\hline numpy, scipy & Scientific \& technical computing \\
\hline pandas & Data manipulation \& aggregation \\
\hline mlpy, scikit-learn & Machine learning \\
\hline theano, tensorflow, keras & Deep learning \\
\hline statsmodels & Statistical analysis \\
\hline nitk, gensim & Text processing \\
\hline networkx & Network analysis \& visualization \\
\hline bokeh, matplotlib, seaborn, plotly & Visualization \\
\hline beautifulsoup, scrapy & Web scraping \\
\hline
\end{tabular}

Python Libraries for Data Science

- NumPy - introduces objects for multidimensional arrays and matrices, as well as functions that allow to easily perform advanced mathematical and statistical operations on those objects and provides vectorization of mathematical operations on arrays and matrices which significantly improves the performance or many other python libraries are built on NumPy

- SciPy - is collection of algorithms for linear algebra, differential equations, numerical integration, optimization, statistics and more

- Pandas - adds data structures and tools designed to work with table-like data (similar to Series and Data Frames in R) and provides tools for data manipulation: reshaping, merging, sorting, slicing, aggregation etc. also allows handling missing data

- SciKit-Learn Visualization libraries - provides machine learning algorithms: classification, regression, clustering, model validation etc. Is built on NumPy, SciPy and matplotlib 
- matplotlib - is python 2D plotting library which produces publication quality figures in a variety of hardcopy formats. Matplotlib has a set of functionalities similar to those of MATLAB: line plots, scatter plots, bar-charts, histograms, pie charts etc.

Python is a great language for doing data analysis, primarily because of the fantastic ecosystem of data-centric Python packages. Pandas is one of those packages, and makes importing and analyzing data much easier. Pandas is an open source, BSD licensed library that provides high-performance, easy-to-use data structures and data analysis tools for the Python programming language.

The two key data structures in Pandas are Series and DataFrames.

- Series can be understood as a 1 dimensional labelled / indexed array. You can access individual elements of this series through these labels.

- A dataframe is similar to Excel workbook - you have column names referring to columns and you have rows, which can be accessed with use of row numbers. The essential difference being that column names and row numbers are known as column and row index, in case of dataframes.

Series and dataframes form the core data model for Pandas in Python. The data sets are first read into these dataframes and then various operations (e.g. group by, aggregation etc.) can be applied very easily to its columns.

The most important thing in Data Analysis is comparing values and selecting data accordingly. The "==" operator works for multiple values in a Pandas Data frame too. Following examples will show how to compare and select data from a Pandas Data frame. A Data frame is a two-dimensional data structure, i.e., data is aligned in a tabular fashion in rows and columns. We can perform basic operations on rows/columns like selecting, deleting, adding, and renaming.

In Order to select a column in Pandas DataFrame, we can either access the columns by calling them by their columns name.

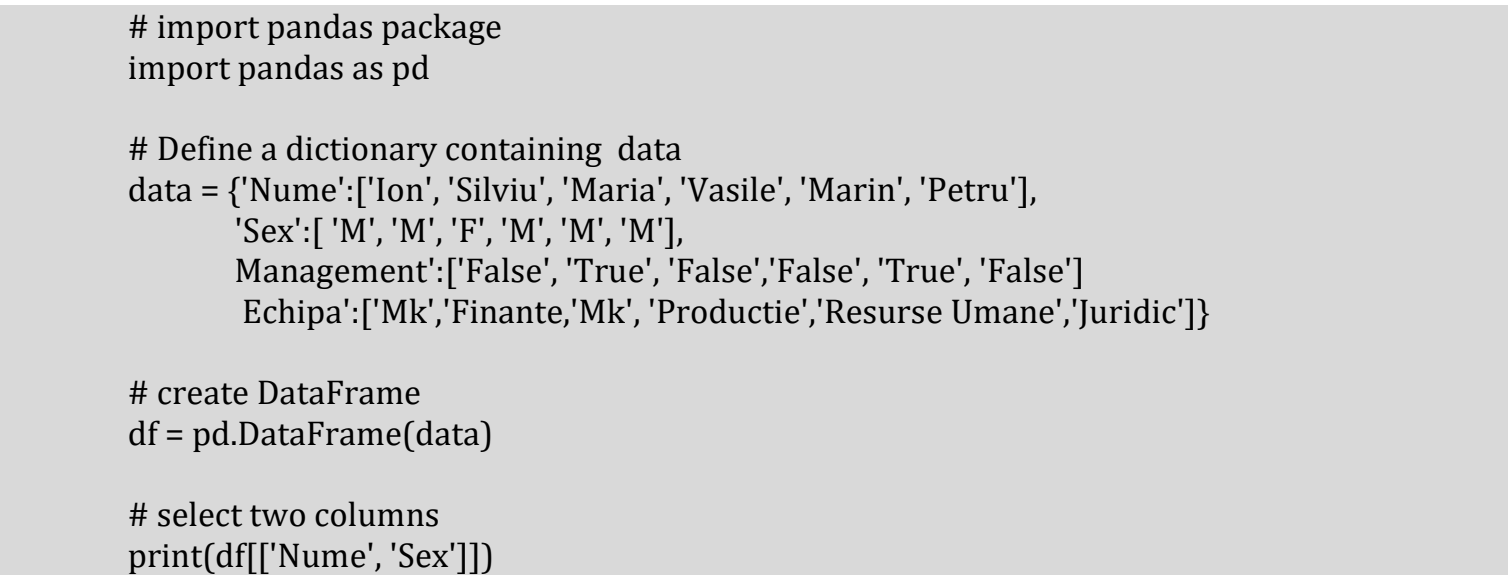

Example 1: Comparing Data - In the following example, in the Sex Column, there are only 2 types of values ("M", " $F$ "). Every row of Sex column is compared to "M" and a boolean series is returned after that.

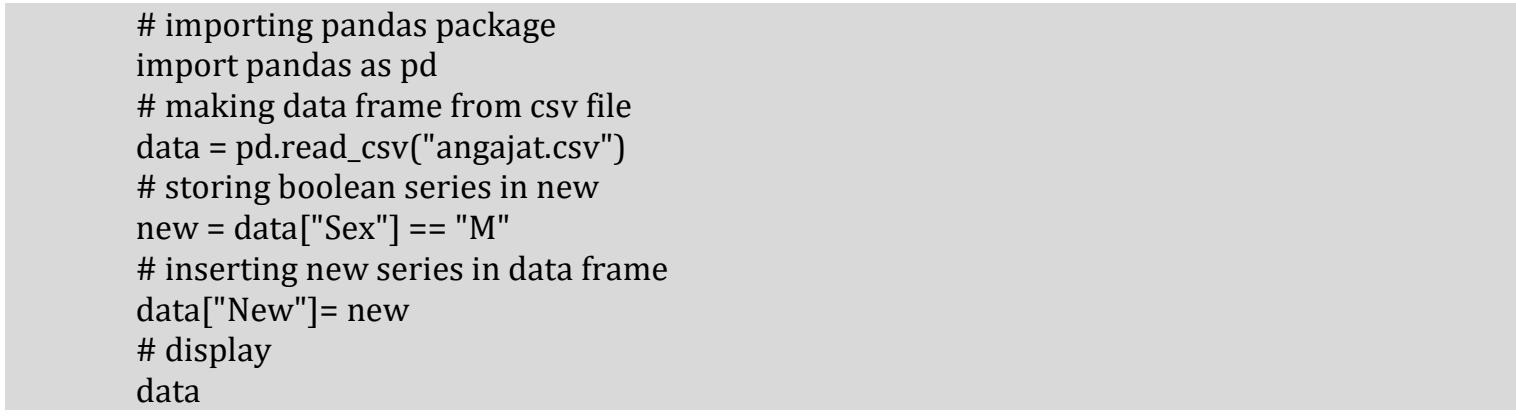

Example 2: Selecting Data - In the following example, the boolean series is passed to the data and only Rows having Sex="M" are returned.

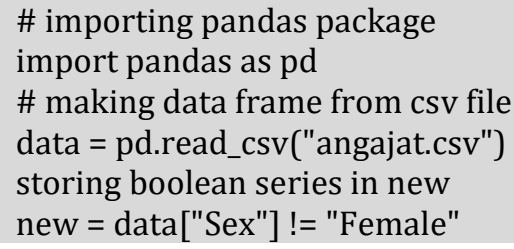




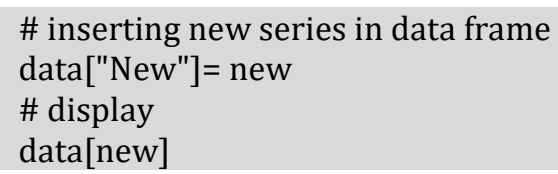

Analyzing data requires a lot of filtering operations. Pandas provide many methods to filter a Data frame and Dataframe.query0 is one of them.

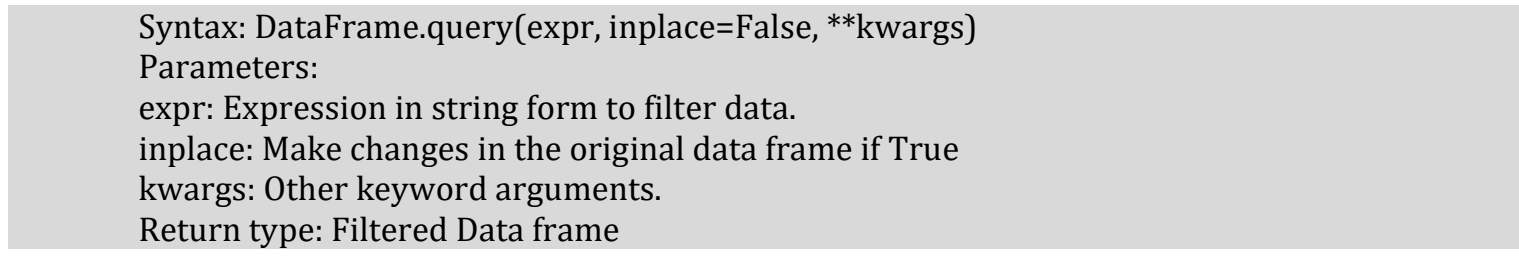

Example 3: Single condition filtering - In this example, the data is filtered on the basis of single condition. Before applying the query 0 method, the spaces in column names have been replaced with '.'.

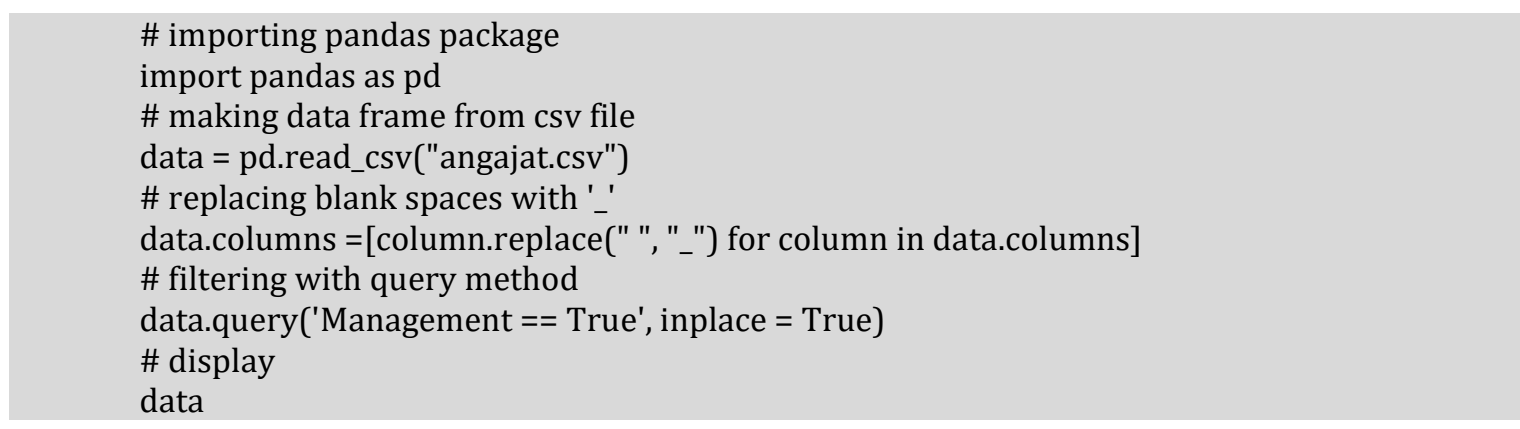

Example 4: Multiple condition filtering - In this example, dataframe has been filtered on multiple conditions. Before applying the query $(0$ method, the spaces in column names have been replaced with '_.'

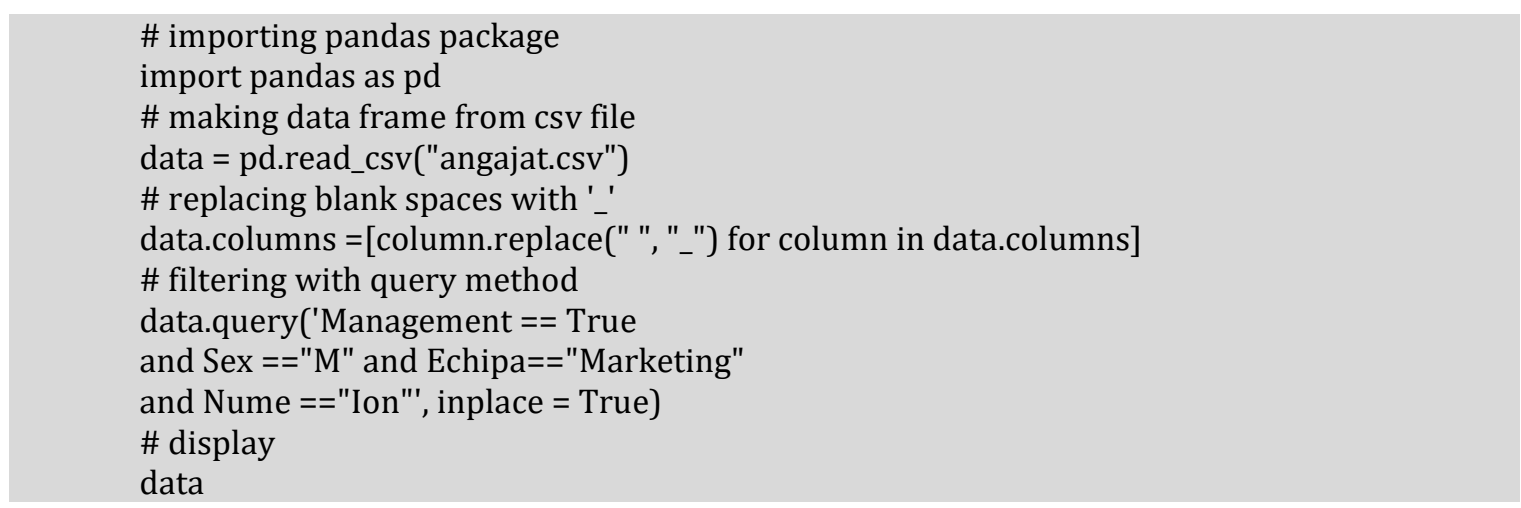

While our exploration of the data, we found a few problems in the data set, which needs to be solved before the data is ready for a good model. This exercise is typically referred as "Data Munging". Here are the problems, we are already aware of:

- There are missing values in some variables. We should estimate those values wisely depending on the amount of missing values and the expected importance of variables.

- We should also look at the non-numerical fields if they contain any useful information.

\section{Acknowledgement}

This work was supported by a grant of the Romanian Ministry of Research and Innovation, CCCDI - UEFISCDI, project number PN-III-P1-1.2-PCCDI-2017-0800 / 86PCCDI/2018 - FutureWeb, within PNCDI III.

\section{Conclusions}

Python has been a great time for munging and preparing data, but less for analyzing and modeling data. Pandas help complete this space by allowing you to perform the entire Python data analysis process without having to go to a domain-specific language such as R. 
Combined with the excellent setof IPython tools and other libraries, the Python data analysis environment excels in performance, productivity and collaboration.

Pandas does not implement significant modeling functions outside linear regression and panes; for that, look at statsmodels. More efforts are still needed to make Python a first-class statistical modeling environment, but we are on the way to this goal.

\section{References}

1. Wes McKinney - Python for Data analysis - O'Reilly Media 2013

2. Guido van Rossum - Tutorial Python ver 2.2 - - 2001

3. www.python.org

4. Wes McKinney\& PyData Development Team - Pandas: powerful Python data analysis toolkit - 2019

5. https://www.geeksforgeeks.org 